\title{
VARIATIONS IN THE LINE SPECTRUM OF \\ A-TYPE SUPERGIANTS
}

\author{
WILLIAM BUSCOMBE \\ Northwestern University, Evanston, Ill., U.S.A.
}

\begin{abstract}
Attention is drawn to a group of stars of luminosity class Ia, in which variation of radial velocity has long been known in semi-regular cycles of 5 to 30 days. The interval of approximate repetition appears to be positively correlated with the luminosity of the star.

Recent spectrophotometry of the absorption lines, especially by Rosendhal, Aydin, Groth, and Buscombe shows a considerable variation in the strength of Balmer lines (with emission components detected at $\mathrm{H} \alpha$ ), as well as those of metallic ions. The microturbulence parameter derived from curves of growth for $\mathrm{Fe}$ II varies by a factor of two or more during some cycles.
\end{abstract}

\section{DISCUSSION}

Hutchings: From many spectra of 6 Cas taken during 1971-72 at Victoria, we find no indication of the radial velocity variations which Abt found in 1957.

Bell: Does the turbulence remain constant on your plates?

Hutchings: We have not obtained microturbulent velocities for all the spectra, but the few we have do not show much variation.

Buscombe: Perhaps I could just add that on the Radcliffe plates, as well as my own from Stromlo, it seemed that the strength of $\mathrm{H} \gamma$ in absorption is well correlated with the microturbulence parameter and ranges over a factor of 2 .

Underhill: Supergiants are very complex and it will be difficult to make model atmospheres. For instance, $\eta$ CMa, B51a, shows evidence for a stationary circumstellar shell and a layer which is moving outward with a velocity of $100 \mathrm{~km} \mathrm{~s}^{-1}$. How are you going to combine these in one model in hydrostatic equilibrium?

Buscombe: When I corresponded with Dr Thackeray, he urged me to take account of the fact that this Carina supergiant is the only one of the southern stars in my program which is involved in visible nebulosity and, indeed, the other three stars seem to be stable and constant in all respects. 